\title{
The Application of Dual Beam FIB Techniques to the Preparation of TEM Sections from Heterogeneous Carbonaceous Chondrites
}

\author{
L. J. Chizmadia ${ }^{*}$, Y. Xu ${ }^{* *}$, C. Schwappach ${ }^{* *}$ and A. J. Brearley ${ }^{* * *}$ \\ *Institute for Astronomy, University of Hawai’i, Honolulu, HI 96822 1chiz@ifa.hawaii.edu \\ ** Intel Corporation, Rio Rancho, NM 87124 \\ *** Department of Earth and Planetary Sciences, University of New Mexico, Albuquerque, NM 87131
}

$\mathrm{CM}$ carbonaceous chondrites have experienced extensive aqueous alteration, prior to their arrival on Earth. The location of this alteration is controversial: models that involve alteration before (preaccretionary alteration) or after (parent-body alteration) the accretion of the final parent asteroid have been proposed. Detailed studies of altered chondrule glass and the corresponding fine-grained rims were conducted on two unbrecciated $\mathrm{CM}$ chondrites that experienced differing degrees of alteration, Y791198 and ALH81002. A comparison of these two meteorites demonstrates that elemental exchange had occurred between the chondrules and their rims [1]. In addition, the presence of secondary Fe-rich and $\mathrm{Ca}$ - and P-rich layers at the rim-chondrule interfaces also indicates closed system behavior between the chondrules and their rims [2]. Both of these observations point to the parent-body alteration model. However, micron-sized Fe,Ni metal grains have been observed by SEM [3] and were determined to be pristine (i.e. unaltered). The presence of pristine Fe,Ni metal grains juxtaposed against hydrated rim materials in Y-791198 has been cited as conclusive evidence that unaltered materials must have been mixed into the fine-grained dust that formed rims after aqueous alteration but before accretion of the final parent asteroid. Therefore, it is important to establish whether the Fe,Ni metal grains are pristine or show evidence of alteration at the micron to submicron scale.

In an attempt to shed light on the pre-accretionary vs. parent-body alteration controversy, we studied $\mathrm{Fe}, \mathrm{Ni}$ metal grains embedded within fine-grained rims around chondrules in Y-791198 using TEM. During routine TEM investigation of samples prepared by conventional ion beam milling, several Feand Ni-bearing grains were observed but were too thick to obtain diffraction patterns to confirm that the grains are metallic Fe,Ni. After the investigation of several electron transparent regions, it became clear that ion milling was inadequate for preparing the potential metal grains for detailed analysis. Therefore, two additional site-specific TEM samples (containing three metal grains from two different finegrained rims) were prepared using focused ion beam (FIB) techniques at Intel Labs, New Mexico.

First, the Fe,Ni metal grains were mapped with secondary electrons (SE) using a field-emission gun SEM. The region of interest was then coated with a thin layer of Pt for protection. A wafer of the sample, containing the Fe,Ni metal grain of interest, was then cut out with a focused $30 \mathrm{kV} \mathrm{Ga}{ }^{+}$ion beam. The wafer was then carefully removed from the thin section with an Omni probe micromanipulator and set upon a continuous carbon film that had been deposited on a $\mathrm{Cu}$ TEM grid (Fig. 1). The first sample was cautiously prepared while the second was aggressively thinned. Further details about this preparatory technique can be found in [4-5]. The samples were then examined at the University of New Mexico using a JEOL JEM2010F field-emission gun high-resolution analytical electron microscope, operating at $\sim 200 \mathrm{kV}$. Multiple TEM techniques including bright and dark field imaging, HRTEM, HAADF-STEM and EFTEM were applied to the samples. 
Three FIB-prepared metal grains $(\sim 4 \mu \mathrm{m}, \sim 4.5 \mu \mathrm{m}$ and $\sim 1.6 \mu \mathrm{m}$ in diameter) were studied. These studies demonstrate conclusively that the Fe,Ni metal grains show clear evidence of alteration at the sub- $\mu \mathrm{m}$ level. BF-TEM images show that the metal grains have a sharp contact with the hydrated rim materials, nanocrystalline serpentine, and have embayments on their edges. Z-contrast STEM imaging shows that locally the grains have developed reentrant features, a few tens of $\mathrm{nm}$ in depth (Fig. 2) similar to Fe metal that has experienced pitting corrosion [e.g. 6-7]. These reentrant features have a lower average $\mathrm{Z}$ than the rest of the metal grain and show an O-enrichment in EFTEM maps that is consistent with local oxidation of the metal grain (Fig. 3, see top right of the interface). However, the amount of alteration is slight so that it is not clear whether it occurred on the parent-body or in the Antarctic ice.

This research was supported by a NASA Cosmochemistry grant (NAG5-13046, A. J. Brearley, P. I.) and by the Intel Corporation.

\section{References}

[1] L. J. Chizmadia and A. J. Brearley LPSC XXXV (2004) CDROM\#1753

[2] L .J. Chizmadia and A. J. Brearley MAPS 67 (2004) abstract\# 5126

[3] K. Metzler, A. Bischoff and D. Stöffler GCA 56 (1992) 2873

[4] P. J. Heaney, E. P. Vicenzi, L. A. Giannuzzi and K. J. T. Livi Am. Min. 86 (2001) 1094

[5] Y. Xu and C. Schwappach Ind. App. Elect. Micro. 6 (2002) 153

[6] G. W. Whitman, R. P. Russell and V. J. Altieri Ind. \& Engr. Chem. 16 (1924) 665

[7] C. Dillon Corrosion Control in the Chemical Process Industries McGraw-Hill Inc. New York 1994

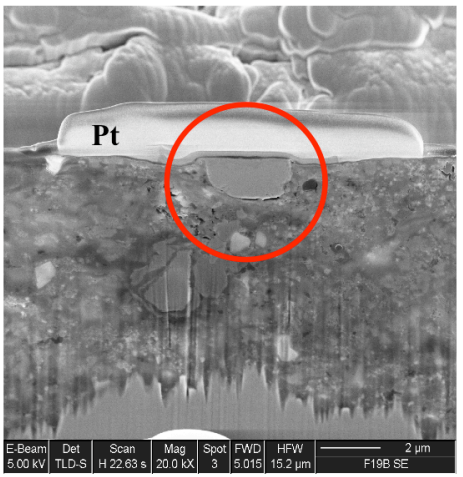

Figure 1. Low magnification SE image of Fe,Ni metal grain FIB- prepared TEM section of a fine-grained rim in Y-791198.

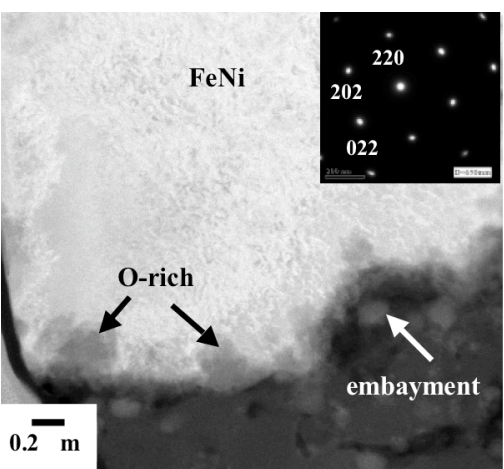

Figure 2. DF-TEM image of an $\mathrm{Fe}, \mathrm{Ni}$ metal grain in a FIB-prepared TEM section of Y-791198.
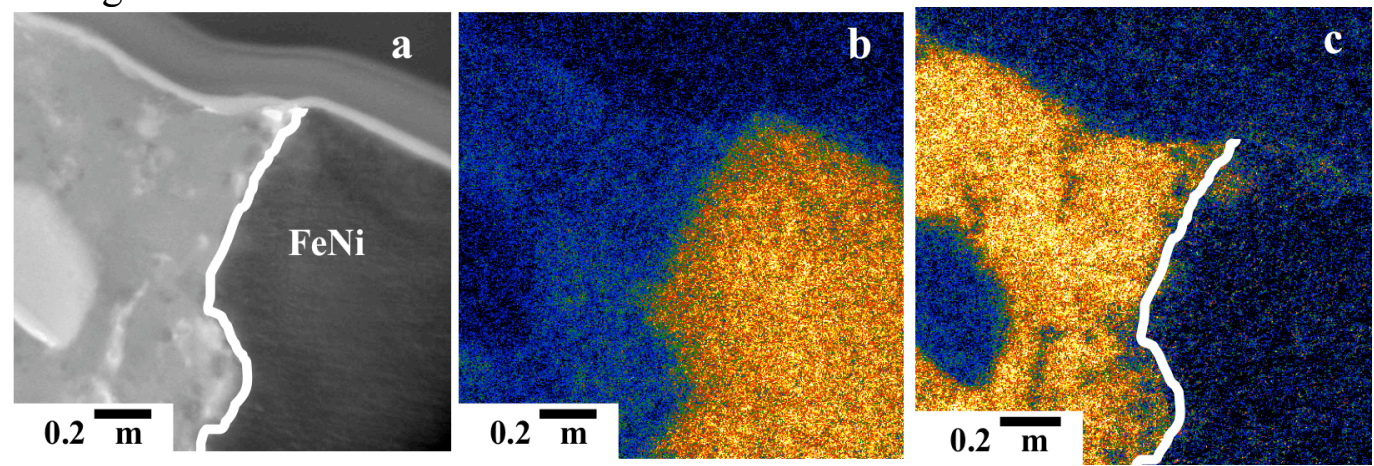

Figure 3. EF-TEM images showing the interface between the metal grain and the other rim materials; a) BF-TEM image, b) Fe-map and c) O-map. Note O-enrichment in metal grain along white line. 